\title{
Validation of GNSS Multipath Model for Space Proximity Operations Using the Hubble Servicing Mission 4 Experiment
}

\author{
B. W. Ashman ${ }^{\dagger}$, J. L. Veldman ${ }^{\ddagger}$, P. Axelrad ${ }^{\ddagger}$, J. L. Garrison*, L. B. Winternitz ${ }^{\dagger}$ \\ ${ }^{\dagger}$ Goddard Space Flight Center, Greenbelt, MD \\ ${ }^{\ddagger}$ University of Colorado Boulder, $\mathrm{CO}$ \\ * Purdue University, West Lafayette, IN
}

\section{BIOGRAPHY}

Benjamin W. Ashman is an engineer in the Navigation and Mission Design Branch (Code 595) at the National Aeronautics and Space Administration (NASA) Goddard Space Flight Center. He received his Ph.D. in Electrical Engineering from Purdue University in 2016 and his B.S. in Electrical Engineering from Ohio University in 2010.

Jeanette L. Veldman completed an M.S. in Aerospace Engineering Sciences at University of Colorado Boulder in 2016. Her research focuses on multipath modeling for space applications. Jeanette received her B.A. in Physics and Mathematics from Central College in 2013.

Penina Axelrad is Professor and Chair of Aerospace Engineering Sciences at the University of Colorado Boulder. She has been involved in GPS related research since 1986. Dr. Axelrad is a Fellow of the ION and the AIAA, and a member of the National Space-Based Positioning, Navigation, and Timing Advisory Board.

James L. Garrison is an Associate Professor in the School of Aeronautics and Astronautics at Purdue University. Previously he was employed by the NASA Goddard Space Flight Center in Greenbelt, MD. He has a Ph.D. in Aerospace Engineering Sciences from the University of Colorado Boulder and is the author or co-author of 34 journal articles and 7 US Patents. Recently, he served as the Chair of GNSS+R 2012, an IEEE-NASA co-sponsored conference.

Luke B. Winternitz is an engineer in the Component and Hardware Systems Branch (Code 596) at NASA Goddard Space Flight Center. He has worked at Goddard since 2001 primarily in the development of GPS receiver technology. He holds B.S., M.S. and Ph.D. degrees in Electrical Engineering from the University of Maryland at College Park.

\section{ABSTRACT}

In the rendezvous and docking of spacecraft, GNSS signals can reflect off the target vehicle and cause large errors in the chaser vehicle receiver at ranges below a few hundred meters. It has been proposed that these additional ray paths, or multipath, be used as a source of information about the state of the target relative to the receiver. With Hubble Servicing Mission 4 as a case study, electromagnetic ray tracing has been used to construct a model of reflected signals from known geometry. Oscillations in the prompt correlator power due to multipath, known as multipath fading, are studied as a means of model validation. Agreement between the measured and simulated multipath fading serves to confirm the presence of signals reflected off the target spacecraft that might be used for relative navigation.

\section{INTRODUCTION}

During spacecraft proximity operations, Global Navigation Satellite System (GNSS) navigation can be corrupted by reflected signals. Throughout docking, for instance, a GNSS receiver on the controlled, chaser spacecraft will receive delayed, attenuated replicas of the direct signals due to reflections off the passive, target spacecraft. This is a source of error known as multipath. Properties of the reflected signals are related to the relative range, orientation, and structure of the reflecting objects and may be used as a source of information about the receiver's environment. The work presented here characterizes reflected signals according to their amplitude, code phase, and carrier phase relative to the corresponding direct signals. Electromagnetic (EM) ray tracing is used to illustrate how these properties arise from the receiver's environment, and a software receiver is used to show the expected impacts of this simulated multipath on tracking results.

In order to confirm that the EM ray tracing and simulated signals accurately model the relationship between geometry, reflected rays, and tracking observables, it is important to find measurements of multipath that can be made from both simulated and experimental data. Previous work demonstrated the multipath-induced error in the zero crossing of the delay lock loop (DLL) discriminator [1], but here the effect on prompt correlator power is studied. This is shown to be a comparatively robust means of detecting multipath. Oscillation in the prompt correlator power, known as multipath fading, is caused by the changing carrier phase of incoming reflected rays relative to the direct signal. During Hubble Servicing Mission 4 (HSM4), two Global Positioning System (GPS) antennas, 
one right and one left-hand circularly polarized, were used to collect raw, sampled radio frequency (RF) data. HSM4 is used as a case study to explore the potential for relative ranging from reflected signals.

\section{BACKGROUND}

Although the concepts here are broadly applicable to all GNSS, the following discussion will use the specific architecture of the GPS C/A signal on the L1 carrier as an example. The direct signal received from the $i$-th GPS satellite can be written

$$
\begin{array}{r}
y^{i}(t)=a^{i} d^{i}\left(t-\tau_{\text {code }}^{i}(t)\right) p^{i}\left(t-\tau_{\text {code }}^{i}(t)\right) \times \\
\cos \left(2 \pi f_{L 1} t+\theta^{i}(t)\right)+v^{i}(t) .
\end{array}
$$

Dropping the satellite superscripts when there is no risk of ambiguity, the signal essentially consists of two binary sequences, $d(t)$ and $p(t)$, modulated by an RF carrier with amplitude $a$. The $d(t)$ sequence represents the 12.5 minute, $50 \mathrm{~Hz}$ navigation data message, and the spreading code $p(t)$ is the $1.023 \mathrm{MHz}$ Coarse Acquisition (C/A) or pseudo-random noise (PRN) code. The code terms arrive at the receive antenna delayed by $\tau_{\text {code }}(t)$ seconds due to the time of propagation.

The carrier frequency of the GPS L1 signal, $f_{L 1}$, is $1.57542 \mathrm{GHz}$. The carrier has phase $\theta(t)=-2 \pi f_{L 1} \tau_{c a r r}(t)+\theta_{0}$, where $\tau_{\text {carr }}(t)$ is the propagation delay of the carrier terms and the initial carrier phase at some reference time $t_{0}$ is $\theta_{0}=-2 \pi f_{L 1} t_{0}$. Random noise affecting the signal at reception is modeled by adding a circular symmetric Gaussian random variable $v(t)$ with zero mean and variance $\sigma^{2}$.

When a large object is located near the receiver, reflections of the GPS signals may also be received. These delayed, attenuated replicas of the direct signals contribute erroneous time of travel information to the receiver's position calculation. Multipath modeling efforts fall into two broad categories: those that seek to model the receiver errors produced by multipath (e.g., [2], [3], and [4]), and those that seek to model properties of the multipath signals themselves (e.g., [5], [6], [7], and [8]). This latter category is most relevant to the work presented here; in order to estimate environmental features from multipath (such as the distance of a reflecting target spacecraft from a receiver), it is necessary to characterize the relationship between the environment features of interest and the observable multipath signal properties.

Ignoring the navigation message and satellite superscripts, the received GPS signal with multipath can be written

$$
\begin{aligned}
y(t)=\sum_{m=0}^{M} a_{m} p\left(t-\tau_{\text {code }, m}(t)\right) \times \\
\cos \left(2 \pi f_{L 1} t+\theta_{m}(t)\right)+v_{m}(t) .
\end{aligned}
$$

for $M$ multipath rays. The carrier phase of the $m$-th ray is $\theta_{m}(t)=-2 \pi f_{L 1} \tau_{c a r r, m}(t)+\theta_{0, m}$. The direct signal is assigned the index $m=0$, while the reflected signals are indexed from $m=1$ to $M$. The direct signal is right-hand circularly polarized (RCP) so, assuming an RCP antenna, the signal amplitude for $m=0$ is a function of $G_{R}$, the antenna's co-polarization gain,

$$
a_{0}=\sqrt{\mathcal{P}_{T, 0} G_{R} \lambda_{L 1}^{2} / 4 \pi}
$$

where $\mathcal{P}_{T, 0}$ is the power spatial density produced by the GPS satellite at the receiver along the line of sight, and $G_{R}$ depends on the elevation and azimuth of the incoming signal. Notation indicating the time dependence of $a_{0}, \mathcal{P}_{T, 0}$, and $G_{R}$ is left off for simplicity. The signal amplitude for $m \neq 0$ is a function of $G_{R \times}$, the antenna's cross polarization gain,

$$
a_{m}=\sqrt{\mathcal{P}_{T, m} G_{R \times} \lambda_{L 1}^{2} / 4 \pi} .
$$

Each multipath ray can be characterized by its amplitude, code phase, and carrier phase relative to the direct signal, denoted $\alpha_{m}, \delta_{m}$ and $\psi_{m}$ respectively. The EM ray tracing used in this work characterizes each multipath ray by its relative electric field strength $E_{m}^{2}=\mathcal{P}_{T, m} / \mathcal{P}_{T, 0}$. The relationship between the simulated value and $\alpha_{m}$ is

$$
\alpha_{m}=E_{m} \sqrt{\frac{G_{R \times}}{G_{R}}}=\frac{a_{m}}{a_{0}},
$$

where again the antenna gains are azimuth and elevation dependent. Relative code phase is $\delta_{m}(t)=c\left(\tau_{\text {code, } m}(t)-\tau_{\text {code }, 0}(t)\right)$ and relative carrier phase $\psi_{m}(t)=\theta_{0}(t)-\theta_{m}(t)$.

As the excess path length traveled by a reflected signal changes, the relative carrier phase will cycle through phases that add to the direct signal constructively and destructively, an effect known as fading. This results in a characteristic oscillation of received power, the frequency of which is determined by the rate of change of the excess path length. Neglecting code and carrier divergence, this relationship can be expressed

$$
\dot{\psi}_{m}(t)=\left(1 / \lambda_{L 1}\right) \dot{\delta}_{m}(t) .
$$

The dot indicates a time derivative. In stationary terrestrial receivers where the reflecting surface is horizontal, this oscillation frequency is known to be a function of satellite elevation [9]. Ground reflections will cause a higher frequency oscillation from low elevation satellites, as the excess path length of the reflections is changing more rapidly than for satellites at higher elevations. In spacecraft docking, the oscillation frequency is driven by the relative geometry of the transmitting GNSS satellite, target, and receiver.

\section{Hubble SERVICING Mission}

The fourth Hubble servicing mission, STS-125, took place in 2009. The crew of the space shuttle Atlantis docked with Hubble Space Telescope (HST) on May 13th and deployed the space telescope on May 18th. During 
rendezvous, docking, and deploy, the Relative Navigation Sensor (RNS) experiment recorded camera imagery from the shuttle cargo bay and estimated the relative position and attitude of HST using several vision processing algorithms [10]. Two GPS antennas were included as part of this experiment and recorded data during the same time frame; one antenna was RCP, intended for receiving direct signals, the other left-hand circularly polarized (LCP), intended for receiving reflected signals. The position and attitude of HST relative to the shuttle was reconstructed for this research from a composite of United Space Alliance's Relative Best Estimate Trajectory (RELBET) product (primarily derived from shuttle-based rendezvous radar measurements) [11] and RNS researcher's image processing estimates. The absolute position of the shuttle was taken from United Space Alliance's Postflight Attitude and Trajectory History (PATH) product [12]. Although the relative state of HST has a $3 \sigma$ position accuracy on the order of centimeters in each dimension, the shuttle position $3 \sigma$ position accuracy is hundreds of meters. Further details on the mission geometry and its reconstruction were given in [13]; new antenna testing results are presented in the following section.

\section{Antenna Properties}

Sensor Systems RCP (Model S67-1575-39) and LCP (Model S67-1575-139) antennas were flown on HSM4. The antennas were mounted in the shuttle cargo bay on top of the Multi-use Logistic Equipment carrier beside the RNS cameras. After initial tracking results suggested poor isolation of direct and reflected signals, it was important to determine the cross-pole discrimination of the antennas. The antennas were not measured prior to the mission, but information provided by Sensor Systems suggested only a $3 \mathrm{~dB}$ cross-pole attenuation [14]. More accurate measurements were needed. The antenna mounting plate was reconstructed according to HSM4 photographs, then the flight antenna gain patterns were measured in the Goddard ElectroMagnetic Anechoic Chamber (GEMAC), shown in Figure 2.

Azimuth cuts of the measured gain patterns are shown in Figure 1. Measurements were taken across antenna elevation from -179 to 179 degrees in increments of one degree at each antenna azimuth cut. Antenna azimuth was tested from 0 to 180 degrees in increments of 15 degrees. Both antennas are active antennas, but the $26 \mathrm{~dB}$ gain of the low noise amplifier has been subtracted. In each case, the co-pole gain is plotted with solid lines and the cross-pole gain with dotted lines. Average gain across the different azimuth cuts is plotted in black; the attenuation of an LCP signal at the RCP antenna boresight is 12 $\mathrm{dB}$, while the attenuation of an RCP signal at the LCP antenna boresight is $7 \mathrm{~dB}$. This is better than the crosspole attenuation specified by the manufacturer, but the poorer polarization of the LCP antenna causes difficulty in the isolation of LCP signals [15].

\section{Simulation}

Sampled GPS data was simulated using MATLAB signal simulation code based on the Goddard Space Flight Center (GSFC) Siggen ("Sig-gen"). Existing code, initially created by GSFC engineers as a development tool, was modified for this research to include multipath. The Advanced GNSS Multipath Model (AGMM) developed at the Colorado Center for Astrodynamics [16] was used to simulate the expected multipath during HSM4. AGMM integrates the commercially-available wave propagation software WinProp ${ }_{\circledast}$ (developed by AWE Communications [17]) into a suite of modules [18]. Three modules were employed to produce the ray tracing results used in this paper: the receiver environment, transmitter motion, and ray tracing modules. Together these modules reproduce the dynamics of HST and the transmitting GPS satellites, then calculate all possible signal paths between the transmitters and receiver based on concepts from geometric optics and the uniform geometrical theory of diffraction. A tenminute ray tracing simulation was developed to replicate HSM4 geometries from 16:22 to 16:32 UTC on May 13, 2009. Continuous interactions were observed lasting up to three minutes. Further detail on the EM ray tracing and signal simulation was presented previously in [13].

\section{Implementation}

Four PRNs, 1, 26, 27, and 30, are used as examples in the following results, each selected for the presence of continuous reflections lasting several seconds. Data simulation and tracking was performed from 16:25:27 to 16:28:12 UTC, a time span of 165 seconds (2.75 minutes). Some practical difficulties arise when using the ray tracing results to simulate signals, however. For example, the delay and power of reflections relative to the direct signal for PRN 26 are shown in Figure 3. It is clear by inspection that a number of reflected rays are arriving simultaneously. This is a common feature of the ray tracing results. When generating simulated signals from these data, however, the individual rays are indistinguishable. It is not possible to associate one time-tagged delay value with a preceding delay value in order to reconstruct the time evolution of a single ray.

To resolve this, a curve fit is used to approximate the reflected ray represented by the data points and an average of the relative power is used.. For instance, in Figure 3 a ray with a slope of $0.0145 \mathrm{~ns} / \mathrm{s}$ starting at a delay of $56.48 \mathrm{~ns}$ would well approximate the reflected signal from 16:26:10 to 16:27:09 UTC. This approach is followed for each of the PRNs, producing the delay profiles described in Table I. This approximation is justified by considering that the plurality of nearly identical, simultaneous reflections is likely due to the necessary discretization in the ray tracing simulation of shapes, such as the curved body of HST, into polygons. The effect can be seen in the ray 

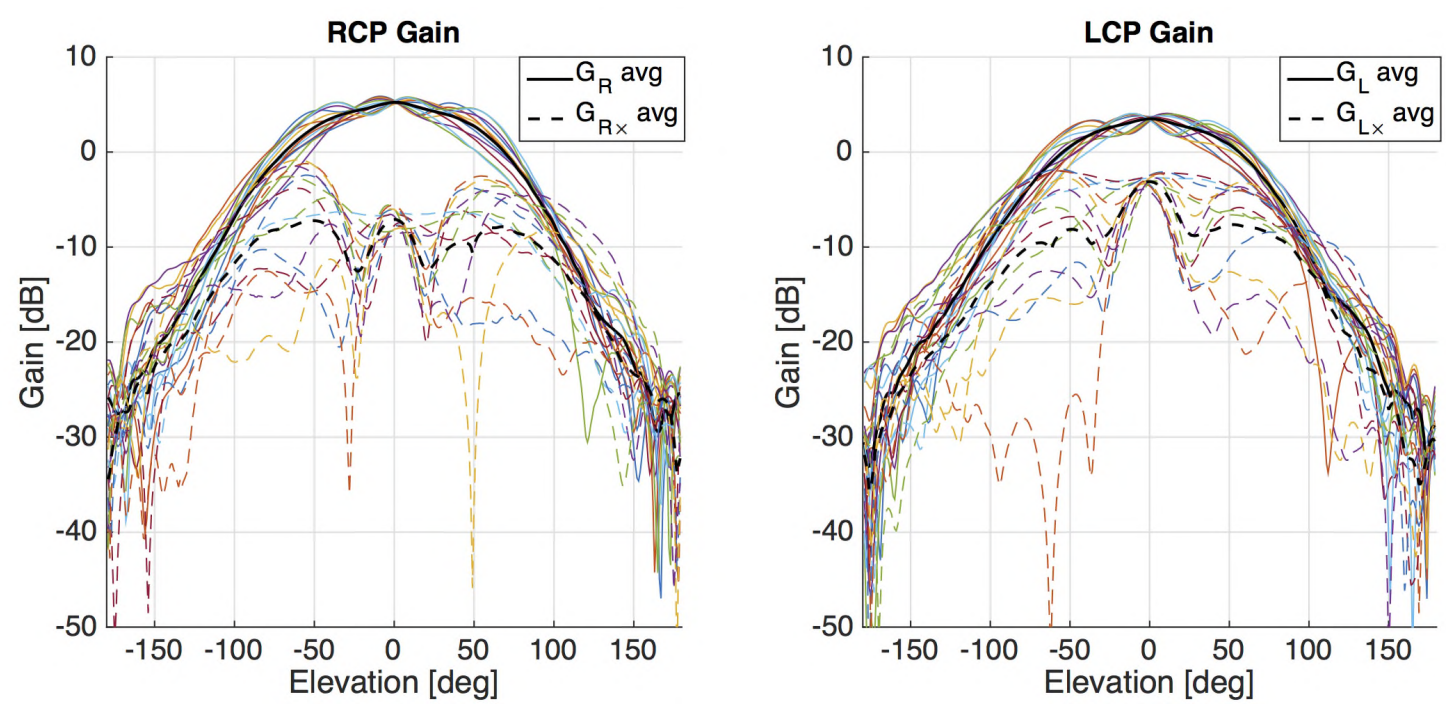

Fig. 1: Elevation (solid) and azimuth (dashed) gain cuts of HSM4 antennas measured in GEMAC

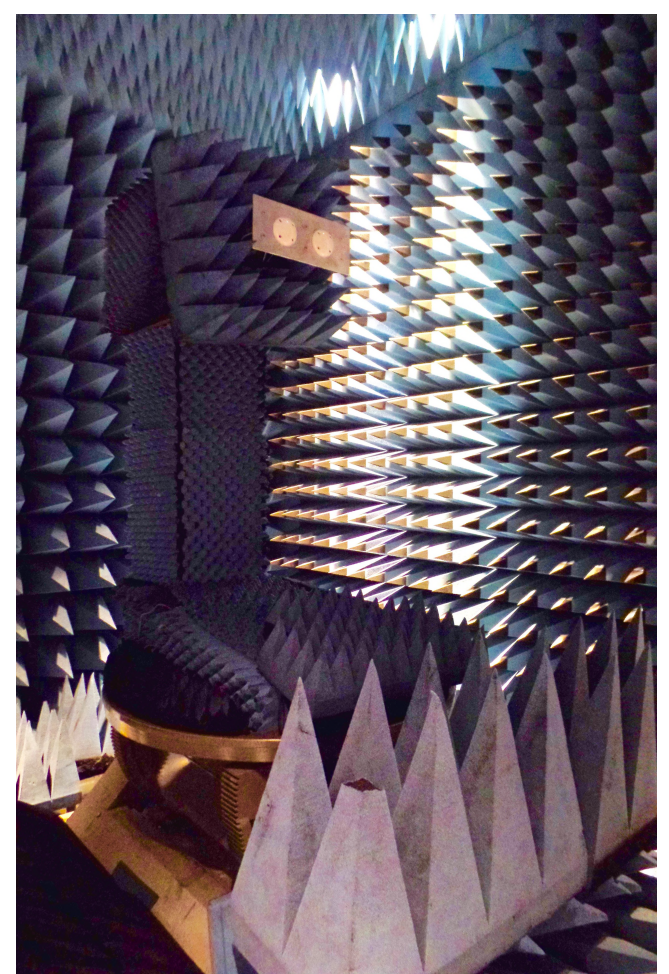

Fig. 2: Antenna testing in GEMAC

tracing simulation itself, shown for a single time instant in Figure 4.

\section{RESULTS}

\section{A. Simulation}

Figures 5 and 6 show the prompt correlator power in the time and frequency domains (left and right plots, respectively). The expected oscillation from the simulated geometry (i.e., $\dot{\delta}$ ) and Equation (6) is given in the $\dot{\psi}$
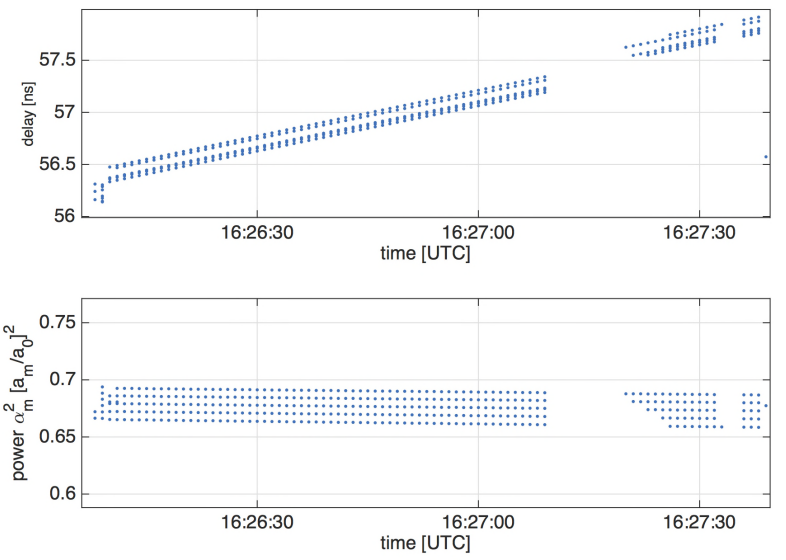

Fig. 3: Relative delay (top) and power (bottom) of reflected rays from PRN 26 ray tracing

Table I: Simulated multipath parameters from ray tracing starting at 16:25:27 UTC

\begin{tabular}{c|c|c|c|c} 
PRN & $\boldsymbol{\delta}_{0}[\mathbf{m}]$ & $\dot{\boldsymbol{\delta}}[\mathbf{c m} / \mathbf{s}]$ & $\boldsymbol{\alpha}_{m}^{2} / \boldsymbol{\alpha}_{0}^{2}$ & $\dot{\boldsymbol{\psi}}[\mathbf{H z}]$ \\
\hline $\mathbf{1}$ & 70.80 & -4.94 & 0.45 & 0.2595 \\
\hline $\mathbf{2 6}$ & 16.75 & 0.44 & 0.66 & 0.0230 \\
\hline $\mathbf{2 7}$ & 16.32 & 0.47 & 0.67 & 0.0246 \\
\hline $\mathbf{3 0}$ & 73.84 & -5.73 & 0.38 & 0.3012
\end{tabular}

column of Table I. Consider PRNs 1 and 30: the peak measured oscillation, determined through the first peak in the frequency domain, is $0.2594 \mathrm{~Hz}$ for PRN 1 and $0.3052 \mathrm{~Hz}$ for PRN 30. Due to the simulated signal length (165 seconds), the resolution of the Fast Fourier Transform (FFT) is $0.0061 \mathrm{~Hz}$; both measured fading frequencies match the expected frequencies to within the FFT resolution.

The multipath oscillations of PRNs 26 and 27 are an 


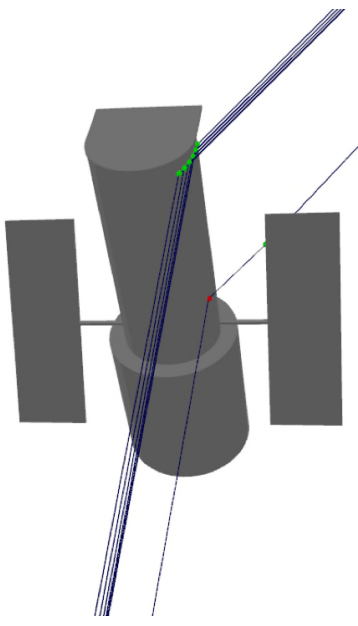

Fig. 4: PRN 26 ray tracing at 16:25 UTC

order of magnitude slower. The frequency of each is difficult to identify in the frequency domain but easily confirmed in the time domain. Figure 7 shows PRN 26, for example, where the calculated frequency is 0.0230 $\mathrm{Hz}$. This corresponds to a period of 43.55 seconds approximately 3.8 periods over the simulation duration. The fading features in Figure 7, measured by tracking simulated data, agree with these values. In the frequency domain on the right, a secondary peak may be present in the spectrum of the filtered power measurement at 0.0244 $\mathrm{Hz}$, but the relatively coarse resolution of the FFT makes this difficult to discern.

\section{B. Experiment}

Multipath-induced oscillation of the prompt correlator power can also be measured in the experimental data from HSM4. The measured frequency of oscillation is generally consistent with the rate of change of the reflected signal's relative delay in Table I.

The conclusion that this oscillation is due to multipath is further supported by the LCP tracking results, shown below the RCP results in both figures. The depth of the oscillation is greater, resulting in a larger frequency domain peak. While the frequency of multipath fading is determined by the rate of change of the multipath delay, the magnitude is a consequence of multipath strength. In the LCP data, reflected signals are expected to be stronger.

The experimentally measured prompt correlator power for PRN 1 is shown in Figure 8. On the top left is the RCP time domain, where a coherent integration time of $1 \mathrm{~ms}$ is used. A $20 \mathrm{~ms}$ non-coherent average is overlaid in orange. The spectra of these unfiltered and filtered RCP power measurements are shown on the top right. The measured frequency of the unfiltered LCP power is $0.2594 \mathrm{~Hz}$ within the FFT resolution of the expected $\dot{\psi}=0.2595 \mathrm{~Hz}$. Frequencies in the filtered RCP and LCP data do not agree quite as closely $(0.2441 \mathrm{~Hz}$ and $0.2686 \mathrm{~Hz}$ respectively). However, the similarity between the results of tracking the simulated and experimental data suggest that the ray specified in Table I for PRN 1 is accurate. PRN 30 exhibits close agreement with ray tracing results as well, shown in Figure 9, with a measured $\dot{\psi}=0.3418 \mathrm{~Hz}$ (from the filtered LCP peak) and calculated $\dot{\psi}=0.3012 \mathrm{~Hz}$.

The slower oscillations of PRNs 26 and 27 are more difficult to detect. A peak of $0.0244 \mathrm{~Hz}$ was measured in the filtered RCP spectra of both PRN 26 and 27, shown in Figures 10 and Figures 11 respectively. LCP tracking fails for PRN 27 and is not shown. While this $\dot{\psi}$ is within the FFT resolution of the calculated frequency for both $(0.0230 \mathrm{~Hz}$ for PRN 27 and $0.0244 \mathrm{~Hz}$ for PRN 26), the peak is not pronounced and the coarseness of the FFT leaves some doubt as to whether this measurement is physically meaningful. The time domain results are inconclusive.

Although the multipath offset rate of change is simulated as constant, this is not the case in reality. Unmodeled variation in the power oscillation frequency over time causes the wider frequency peak observed in PRNs 1 and 30 , as compared to the simulated cases, and contributes to the difficulty in measuring $\dot{\psi}$ experimentally for PRNs 26 and 27.

\section{CONCLUSIONS}

Multipath fading confirms the presence of reflected signals in the HSM4 data. The general agreement of these features with the effects of simulated Hubble-reflected signals provides validation that the multipath model correctly represents the GNSS reflections received during docking. Consequently, ray tracing results support the hypothesis that there are reflections off Hubble that may be useful for relative navigation. Although the multipath fading frequency provides only a range rate measurement, other measurements can be used to extract range from reflected signals. For example, multipath parameters can be estimated from deformation of the code correlation shape (see [5] and [8]) or the reflected signals may be tracked independently. Such measurements rely on sufficiently wide receiver bandwidth and reflected signal isolation (e.g., an LCP antenna with good cross-polarization rejection) respectively [15]. The study of these techniques is ongoing.

\section{ACKNOWLEDGMENTS}

The authors gratefully acknowledge the assistance of engineers at NASA's Goddard Space Flight Center throughout this work, in particular Victor Fontanez, Sam Price and Jenny Donaldson, as well as the help of Dennis Akos at the University of Colorado Boulder. Jeanette Veldman and Penina Axelrad were supported by NASA Goddard Space Flight Center under Cooperative Agreement NNX13AH90A. 

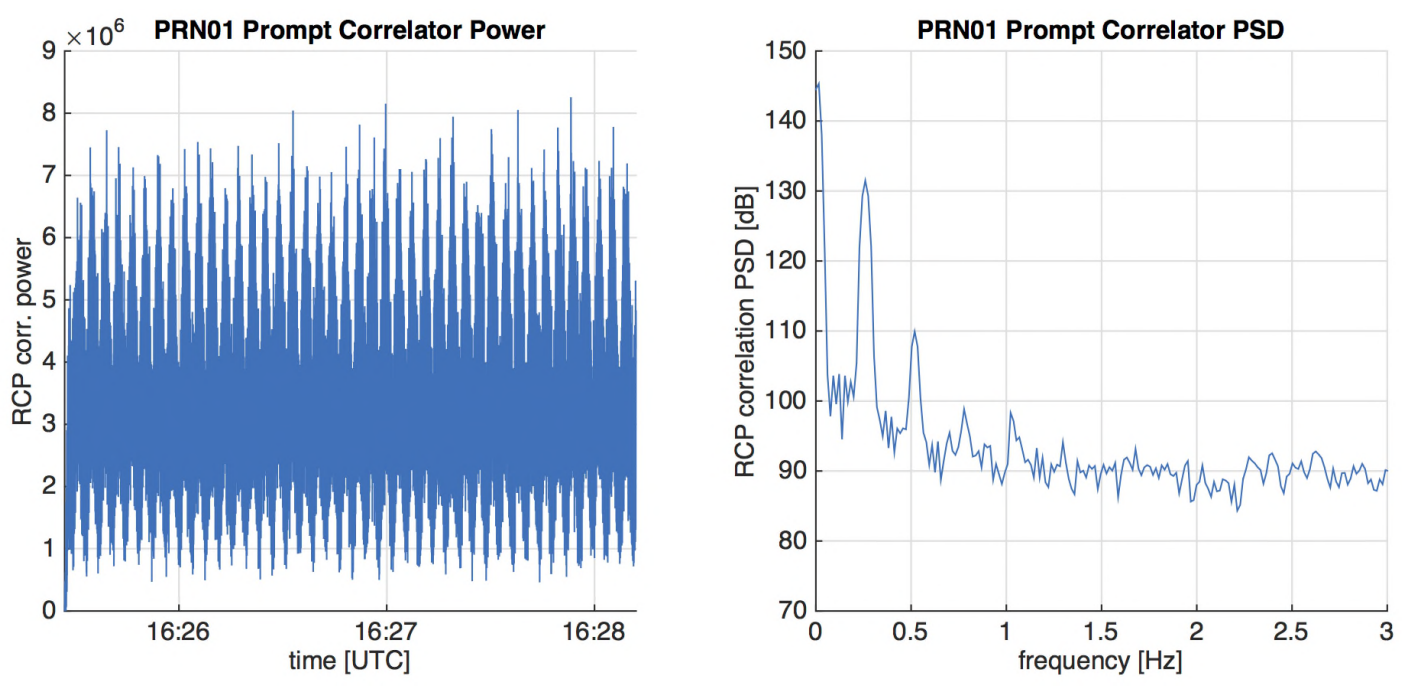

Fig. 5: Simulation: PRN 1 prompt power oscillation
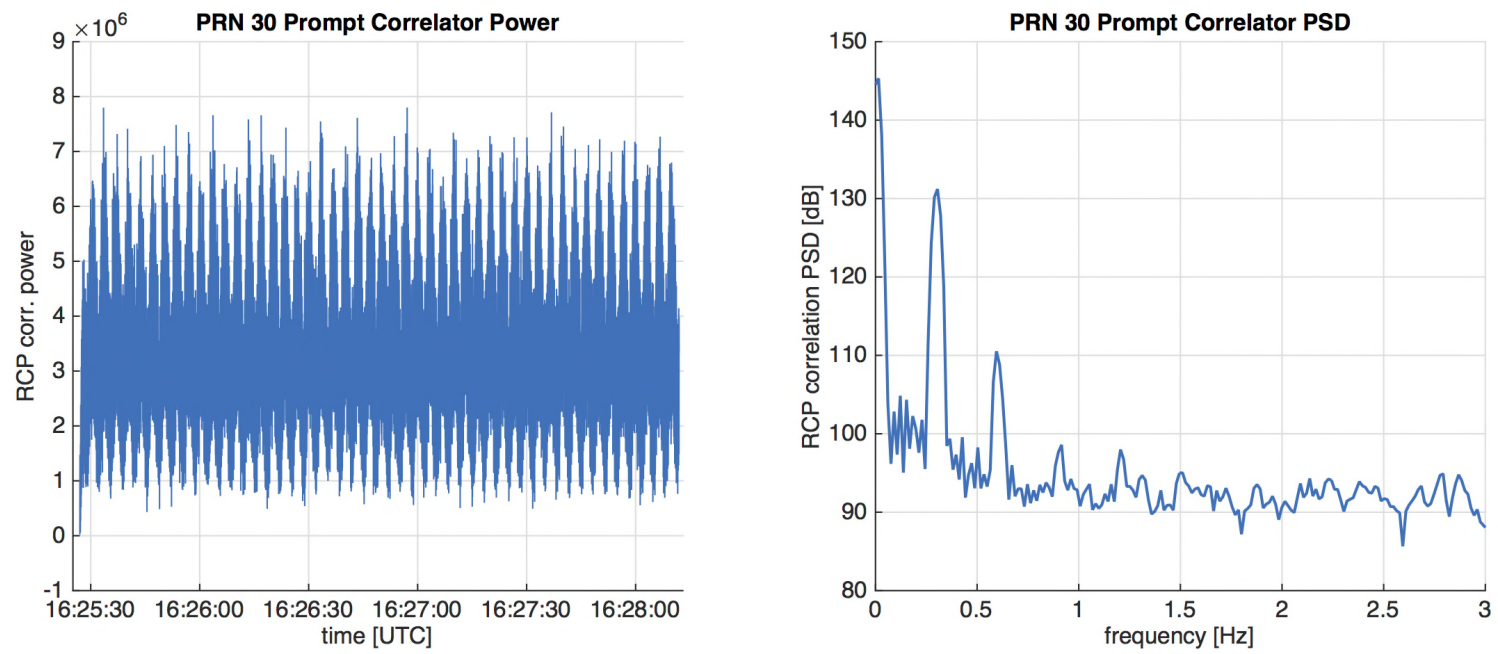

Fig. 6: Simulation: PRN 30 prompt power oscillation

\section{REFERENCES}

[1] Benjamin Ashman and Jeanette Veldman and James Garrison and Penina Axelrad, "Evaluation of the GNSS Multipath Environment in Space Proximity Operations: Experimental and Simulation Studies of Code Correlations in Hubble Servicing Mission 4,' in Proceedings of the ION 2015 Pacific PNT Meeting, (Honolulu,HI), pp. 863-871, Institute of Navigation, April 2015.

[2] Sunil Bisnath and Richard Langley, "Pseudorange Multipath Mitigation By Means of Multipath Monitoring and De-Weighting," in Proceedings of the International Symposium on Kinematic Systems in Geodesy, Geomatics and Navigation, (Banff, Alberta), pp. 392 400, June 2001.

[3] M. Powe and F. Zanier and M. Porretta and A. Garcia-Rodriguez and O. Mongrard, "Analysis of the International Space Station Multipath and Masking Environment for Automated Transfer Vehicle Relative GPS Rendezvous Manoeuvres," in 2012 6th ESA Workshop on Satellite Navigation Technologies and European Workshop on GNSS Signals and Signal Processing, (NAVITEC), pp. 1-7, Institute of Electrical and Electronic Engineers, Dec 2012.

[4] D. E. Gaylor and E. G. Lightsey and K. W. Key, "Effects of Multipath and Signal Blockage on GPS Navigation in the Vicinity of the International Space Station (ISS)," Navigation, vol. 52 , pp. 61-70, 2005.

[5] R. D. J. van Nee, Multipath and Multi-Transmitter Interference in Spread-Spectrum Communication and Navigation Systems. $\mathrm{PhD}$ thesis, Delft University of Technology, 1995.

[6] Sung Byun and George Hajj and Lawrence Young, "Development and Application of GPS Signal Multipath Simulator," Radio Science, vol. 37, pp. 10.1-10.23, November 2002.

[7] Marshall E. Haker and John F. Raquet, "Tracking Multipath in Received GNSS Signals through use of a Signal Decomposition and Parameterization Algorithm," in Proceedings of the 25th International Technical Meeting of The Satellite Division of the Institute of Navigation (ION GNSS 2012), (Nashville, TN), pp. 151-156, September 2012.

[8] Mark Psiaki and Tunc Ertan and Brady O'Hanlon and Steven Powell, "GNSS Multipath Mitigation using Antenna Motion," Journal of the Institute of Navigation, vol. 62, pp. 1 -22, Spring 2015.

[9] Kristine Larson and Andria Bilich and Penina Axelrad, "Improving the Precision of High-rate GPS," Journal of Geophysical Research, vol. 112, no. B05422, pp. 1-11, 2007.

[10] Bo Naasz and John Van Eepoel and Steve Queen and Michael 

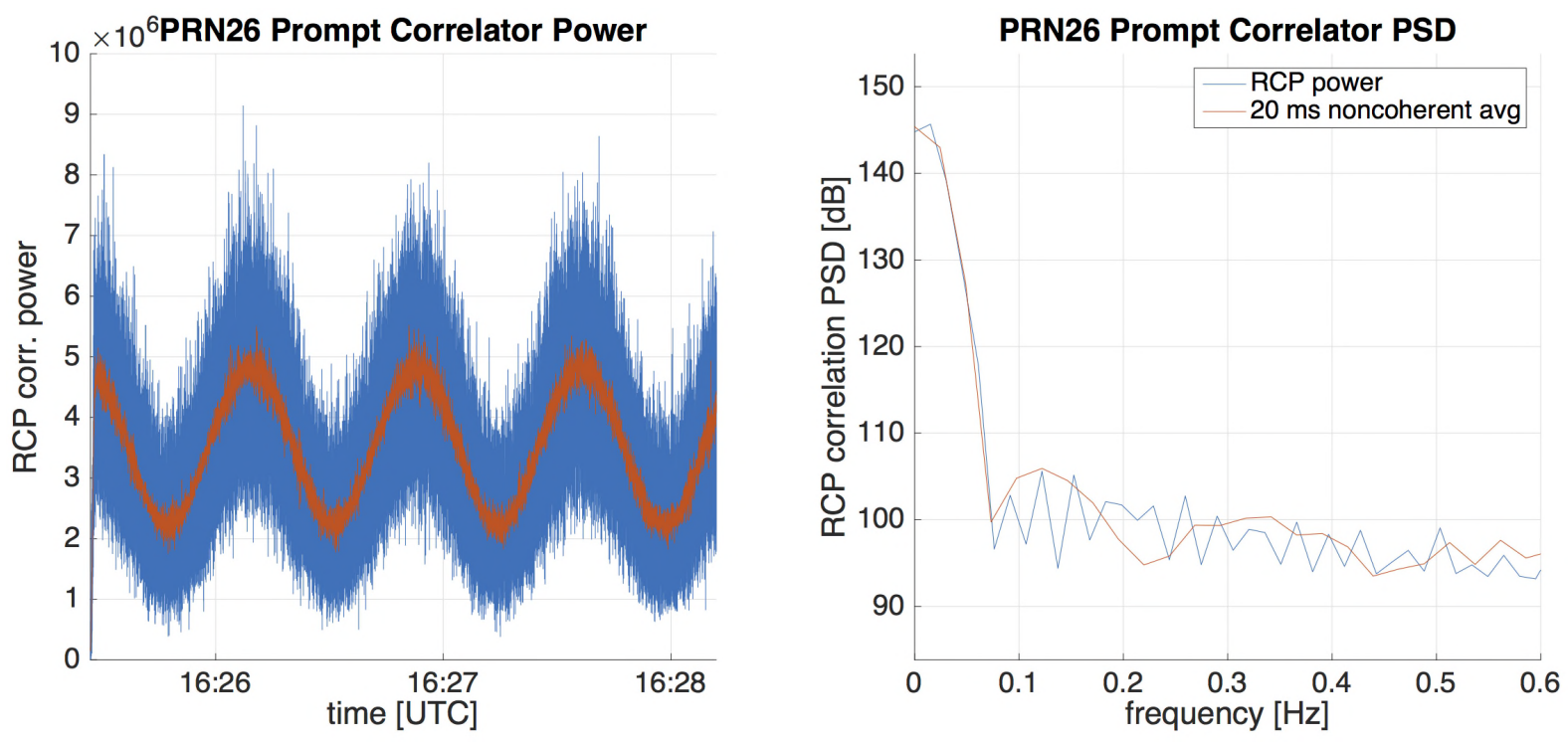

Fig. 7: Simulation: PRN 26 prompt power oscillation
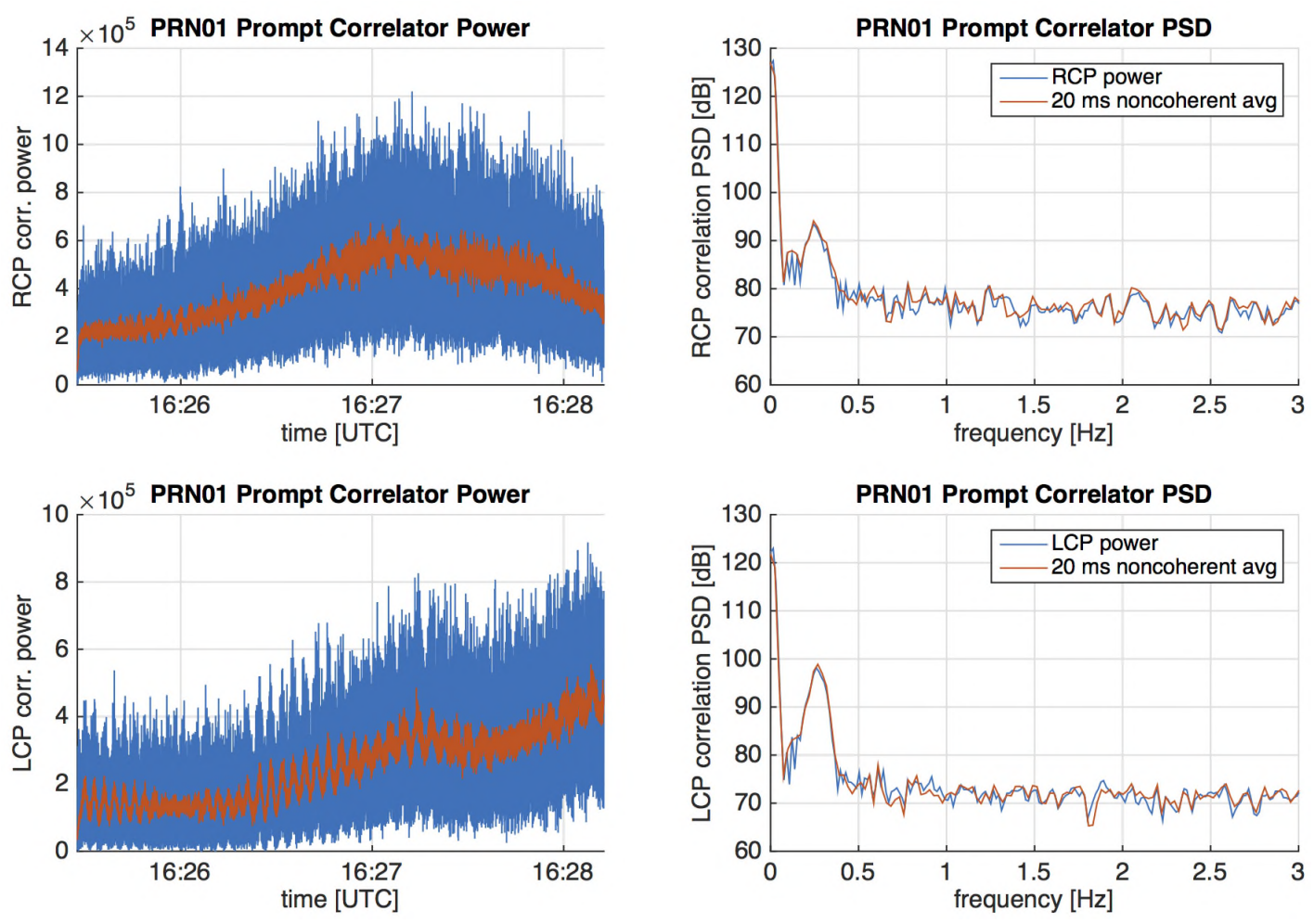

Fig. 8: Experiment: PRN 1 prompt power oscillation 

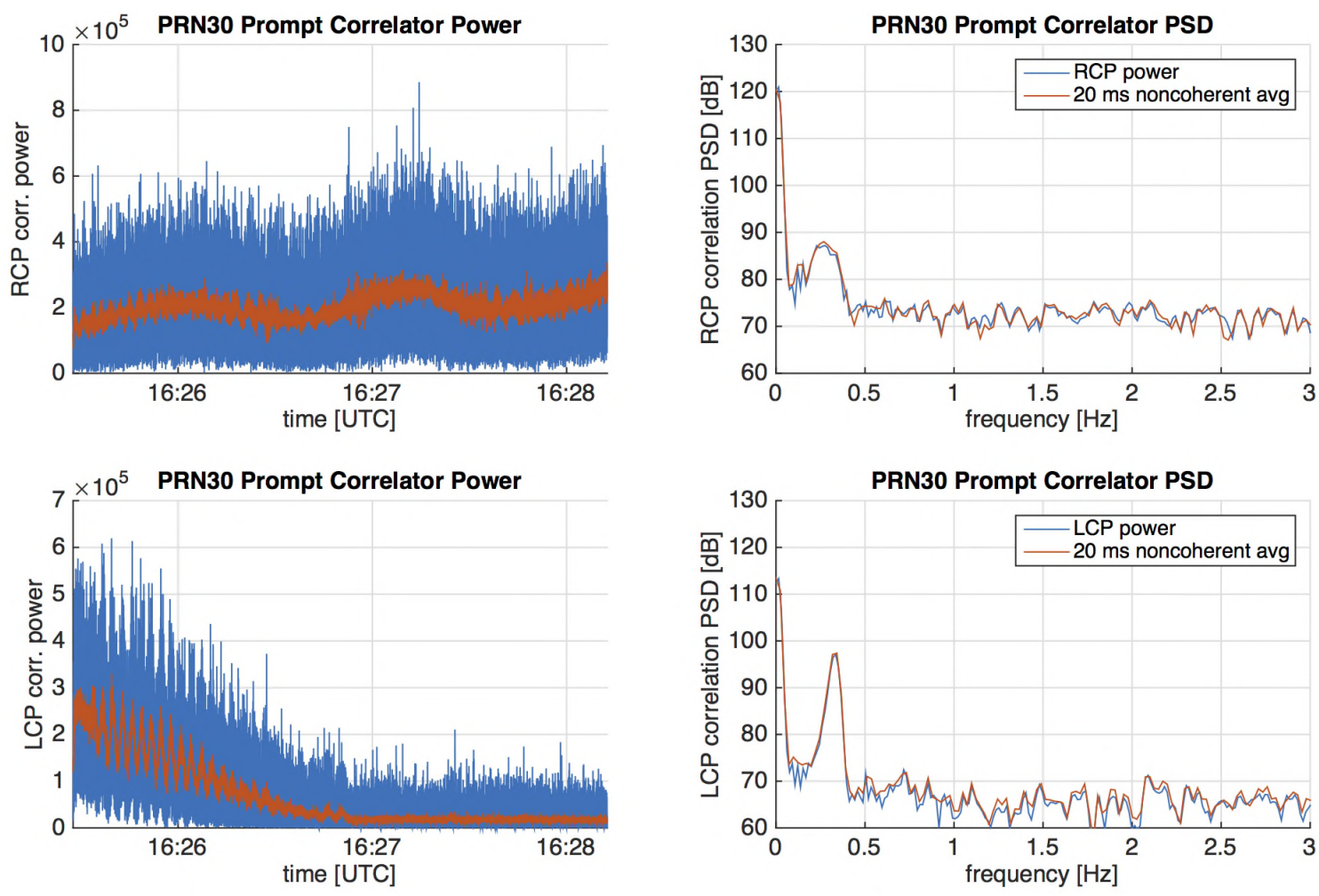

Fig. 9: Experiment: PRN 30 prompt power oscillation
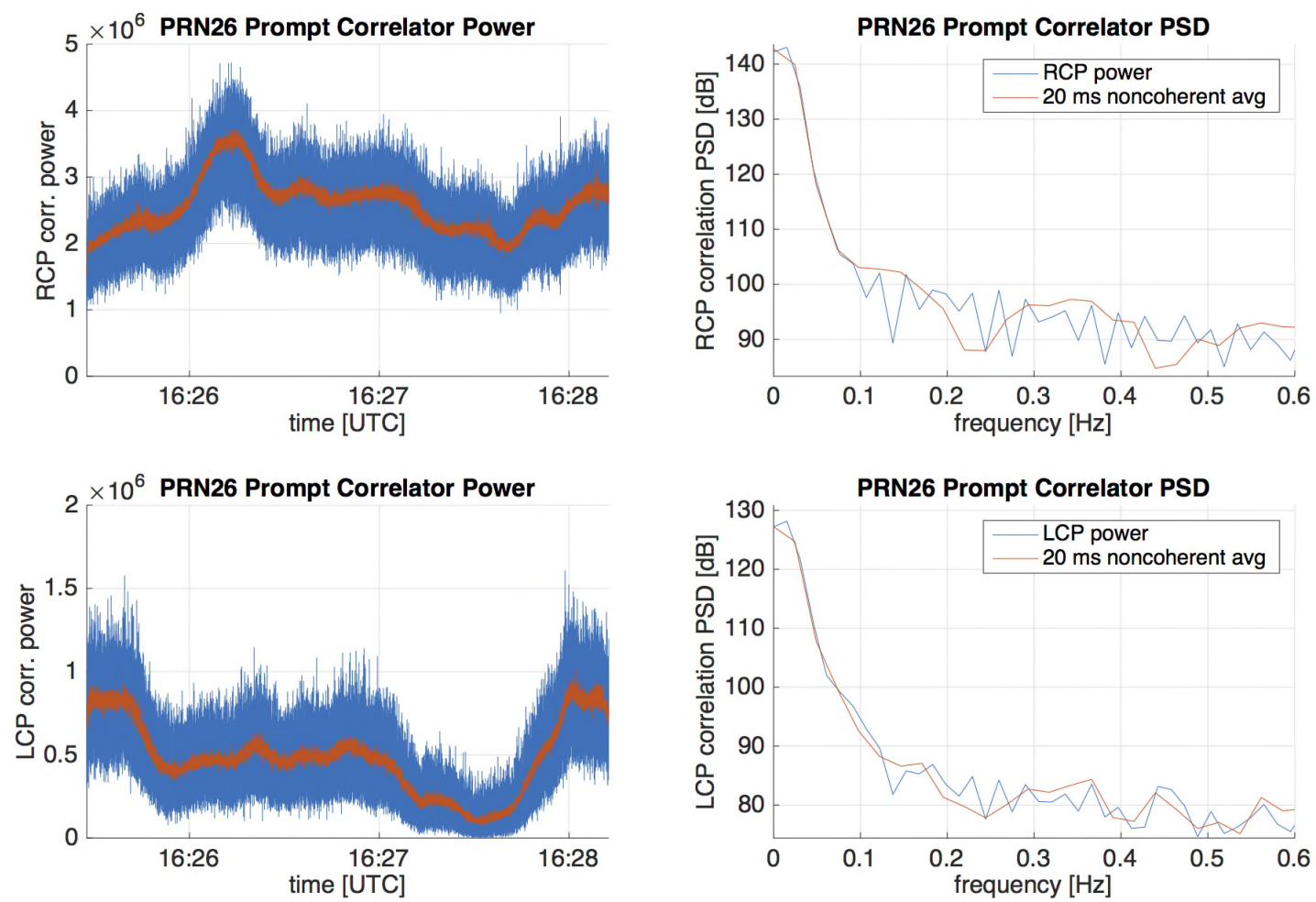

Fig. 10: Experiment: PRN 26 prompt power oscillation 

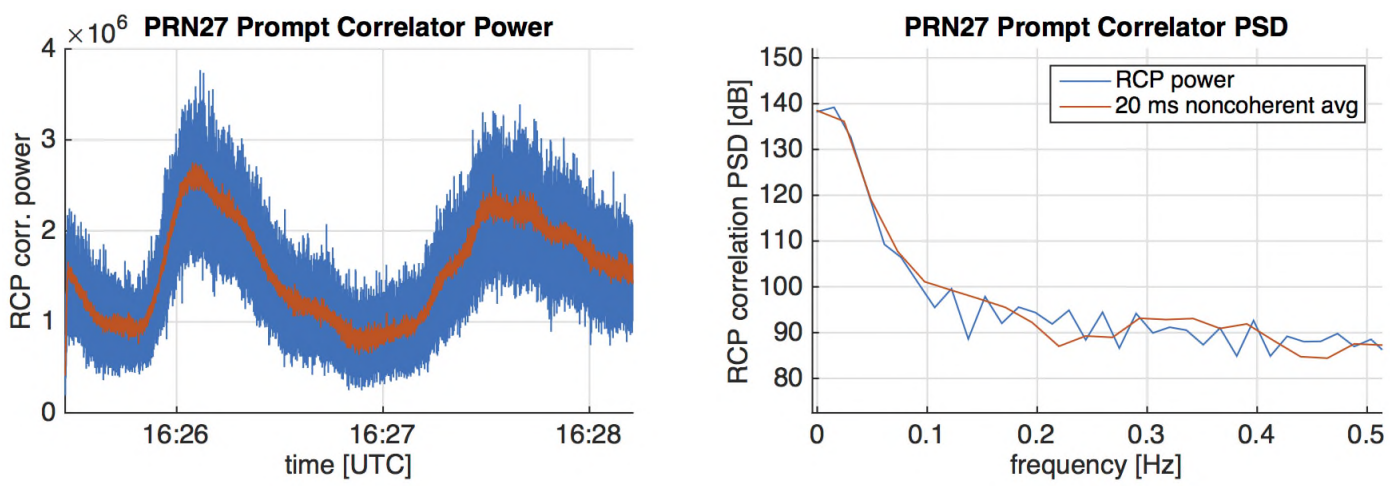

Fig. 11: Experiment: PRN 27 prompt power oscillation

Southward and Joel Hannah, "Flight Results of the HST SM4 Relative Navigation Sensor System," 33rd Annual AAS Guidance and Control Conference, p. 14, February 2010.

[11] Navigation Group of the Flight Design and Dynamics Department, "Internal ICD: RELBET Product," ICD-I-TOP-001, United Space Alliance Company, December 2006.

[12] Navigation Group of the Flight Design and Dynamics Department, "Internal ICD: PATH Product," ICD-I-TOP-002, United Space Alliance Company, April 2007.

[13] Benjamin Ashman and James Garrison, "Tracking of Direct and Reflected Global Navigation Satellite System (GNSS) Signals in Hubble Servicing Mission 4," in Proceedings of the ION 2013 Pacific PNT Meeting, (Honolulu,HI), pp. 1117-1124, Institute of Navigation, April 2013

[14] "GPS S67-1575-39/139 Specifications." Sensor Systems Inc.

[15] B. Ashman, Incorporation of GNSS Multipath to Improve Autonomous Rendezvous, Docking, and Proximity Operations in Space. PhD thesis, Purdue University, 2016.

[16] Jan Weiss and Penina Axelrad and Steve Anderson, "A GNSS Code Multipath Model for Semi-Urban, Aircraft, and Ship Environments," NAVIGATION, vol. 54, no. 4, pp. 294-307, 2007.

[17] AWE Communications, "WinProp Version 12.30," 2012.

[18] J. P. Weiss, Modeling and Characterization of Multipath in Global Navigation Satellite System Ranging Signals. PhD thesis, University of Colorado, 2007. 